INPLASY

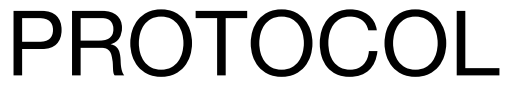

To cite: Wang et al. Efficacy and safety of acupuncture and moxibustion combined with the external application of traditional Chinese medicine in the treatment of primary liver cancer: A systematic review and meta-analysis. Inplasy protocol 2021100029. doi: 10.37766/inplasy2021.10.0029

Received: 09 October 2021

Published: 09 October 2021

Corresponding author:

Tiejun Liu

liutiejun699@163.com

Author Affiliation: Changchun University of Chinese Medicine.

Support: None.

Review Stage at time of this submission: The review has not yet started.

Conflicts of interest:

None declared.

\section{Efficacy and safety of acupuncture and moxibustion combined with the external application of traditional Chinese medicine in the treatment of primary liver cancer: A systematic review and meta-analysis}

Wang, S1; Xiong, Z2; Liu, Y3; Leng, L4; Deng, H5; Shen, D6; Meng, $\mathrm{X}^{7}$; Liu, T8.

Review question / Objective: The aim of this systematic review is to valuate the efficacy and safety of acupuncture and moxibustion combined with the external application of traditional Chinese medicine in the treatment of primary liver cancer. To this end, the proposed systematic review will address the following question: Which is the best choice to effective in improving The short-term curative effect is effective, the quality of life is stable, and the survival rate of patients is half a year/one year in patients with primary liver cancer, acupuncture and moxibustion combined with the external application of traditional Chinese medicine or the best supportive treatment?

Information sources: We will search the following databases: PubMed, Web of Science, GCBI, Embase, OVID, AMED, Cochrane Library, CNKI, VIP, CBM and Wanfang. At the same time, we will manually search all reference lists from relevant systematic reviews to find other eligible studies. We will exclude all conference records, reviews, meta-analyses, newspapers, guides, letters and other documents. When the full text or the required information in the analysis process was missing, the author of the studies was contacted for data.

INPLASY registration number: This protocol was registered with the International Platform of Registered Systematic Review and Meta-Analysis Protocols (INPLASY) on 09 October 2021 and was last updated on 09 October 2021 (registration number INPLASY2021100029).

\section{INTRODUCTION}

Review question / Objective: The aim of this systematic review is to valuate the efficacy and safety of acupuncture and moxibustion combined with the external application of traditional Chinese medicine in the treatment of primary liver cancer. To this end, the proposed systematic review will address the following question: Which 
is the best choice to effective in improving The short-term curative effect is effective, the quality of life is stable, and the survival rate of patients is half a year/one year in patients with primary liver cancer, acupuncture and moxibustion combined with the external application of traditional Chinese medicine or the best supportive treatment?

Condition being studied: Primary liver cancer (PLC) is one of the most common malignant tumors in the world. Its incidence and lethality rank 6th and 3rd, respectively, among malignant tumors in the world. There were between 830,000 and 906,000 new cases of PLC diagnosed in 2020. Between $4.7 \%$ and $8.3 \%$ of new cases worldwide result in death, and this number is increasing year by year, seriously endangering people's lives and health.[1,2] Although various methods such as hepatectomy, liver transplantation, local ablation therapy, and systemic therapy are currently available to treat PLC, their use is often limited by factors such as the size of a patient's tumor, a patient's liver function score, or a patient's general state of health. Therefore, the mortality rate, recurrence rate and overall prognosis of PLC have not improved. $[3,4]$ In short, the health impacts and economic burdens of treating PLC are common problems facing the world population. [5]In recent years, acupuncture combined with the external application of traditional Chinese medicine in the treatment of PLC has been recognized by doctors and patients due to its simplicity and convenience.[6] As an important part of traditional Chinese medicine, acupuncture has been recognized for its effects in improving physical fitness and relieving patients' pain. It has also been used in the treatment and care of PLC and its complications.[7, 8] The external application of traditional Chinese medicine as an adjuvant treatment is characterized by its low cost and direct, topical administration method, bypassing the gastrointestinal tract. These are its obvious advantages. [9,10] However, the effectiveness of acupuncture combined with the external application of traditional Chinese medicine in the treatment of PLC lacks evidence-based support, and the incidence of toxicity and side effects still needs to be further verified; these are both important barriers to its application. Therefore, this systematic review and meta-analysis evaluated the efficacy and safety of acupuncture combined with the external application of traditional Chinese medicine in the treatment of primary liver cancer.

\section{METHODS}

Search strategy: We will search PubMed, Web of Science, GCBI, Embase, OVID, AMED, Cochrane Library, CNKI, VIP, CBM and Wanfang databases. As of September 15, 2021, there are no restrictions on search language, publication time, and publication status. We will use the following medical keywords to search, including: "acupuncture", "external application of traditional Chinese medicine", and "primary liver cancer". At the same time, we will manually search all reference lists from relevant systematic reviews to find other eligible studies.

Participant or population: Patients with liver cancer who have undergone acupuncture and moxibustion combined with the external application of traditional Chinese medicine or the best supportive treatment. There are no restrictions on race, age, gender, etc.

Intervention: Acupuncture and moxibustion combined with the external application of traditional Chinese medicine.

Comparator: The best supportive treatment.

Study designs to be included: Randomized controlled study.

Eligibility criteria: The inclusion criteria are: (1) the type of study is randomized controlled studies (RCTs); (2) the included patients are patients with primary liver cancer; (3) the experimental group receives a combination of acupuncture and external application of traditional Chinese medicine, and the control group receives the best 
supportive treatment (4) Assess the patient's survival, short-term curative effect and efficiency, improvement of quality of life, and occurrence of adverse events. Main outcomes are the short-term curative effect, the improvement of the quality of life, and the half-year and oneyear survival rates of patients.

Information sources: We will search the following databases:PubMed, Web of Science, GCBI, Embase, OVID, AMED, Cochrane Library, CNKI, VIP, CBM and Wanfang. At the same time, we will manually search all reference lists from relevant systematic reviews to find other eligible studies. We will exclude all conference records, reviews, metaanalyses, newspapers, guides, letters and other documents. When the full text or the required information in the analysis process was missing, the author of the studies was contacted for data.

Main outcome(s): The short-term curative effect was measurable, patient quality of life was improved, and the survival rate of patients was between half a year and 1 year.

Additional outcome(s): Additional outcomes included the occurrence of adverse events, such as side effects, nausea and vomiting, skin allergies, and liver damage.

Data management: First, two researchers independently screened and sorted the preliminary data according to the above mentioned inclusion criteria. Then, two other reviewers reviewed and re-evaluated whether the included data met the selection requirements. We will exclude all conference records, reviews, metaanalyses, newspapers, guides, letters and other documents. During the research period, any differences between the researchers' assessments were resolved through research and negotiation with another principal researcher until a consensus was reached. Finally, another researcher resolved any remaining differences, and then a full-text report was obtained. The research selection process is represented as a PRISMA flowchart.[12] When the full text of a study or the required information in the analysis was missing, the author of the study was contacted, and the data were requested. The two authors independently performed data extraction according to the Cochrane manual guidelines and reported the results according to the PRISMA guidelines $[13,14]$. Any differences were resolved by a consensus of all authors.

Quality assessment / Risk of bias analysis: The two authors separately used the Cochrane risk bias assessment tool to assess the quality of the randomized studies.[15] The Cochrane bias risk assessment tool evaluates risk of bias in six domains: selection bias (random sequence generation, allocation hiding), implementation bias, measurement bias, follow-up bias, reporting bias, and other biases. Each item uses low bias and uncertain bias risk. In addition, the high offset is judged and divided. We used Begg's test and set $P<0.1$ as statistically significant, and we used a funnel chart to assess publication bias. When the quality of a single study was evaluated differently by the two authors, the inconsistency was resolved through a consensus among all authors.

Strategy of data synthesis: We used the random effects model in Review Manager software (REVMAN v5.3 Cochrane Collaboration) for the meta-analysis. $P<0.05$ was considered statistically significant. Two authors independently performed data extraction and data input, and the third author checked the data; the first two authors performed the data calculations. The hazard ratio of the $95 \%$ confidence interval, or $95 \% \mathrm{Cl}$, of the binary classification results or the continuous results was evaluated. We used I2 statistics to detect clinical heterogeneity, which was scored as follows: $0 \% \leq 12<25 \%$, no heterogeneity; $25 \% \leq 12<50 \%$, mild heterogeneity; $50 \% \leq 12<75 \%$, moderate heterogeneity; and $12 \geq 75 \%$, severe heterogeneity. If there was a high degree of heterogeneity between trials $(12 \geq 50 \%$ or 
$P<0.1$ ), we tried to determine the source of the heterogeneity through subgroup analysis, meta-regression and sensitivity analysis. Sensitivity analysis was performed by omitting individual studies.

Subgroup analysis: We will use subgroup analysis based on different interventions, controls, and outcomes.

Sensitivity analysis: Sensitivity analysis was performed by omitting individual studies.

Language: There were be no restrictions regarding language.

\section{Country(ies) involved: China.}

Other relevant information: References: 1 Sung H, Ferlay J, Siegel RL, et al. Global Cancer Statistics 2020: GLOBOCAN Estimates of Incidence and Mortality Worldwide for 36 Cancers in 185 Countries. CA: a cancer journal for clinicians 2021;71(3):209-49. 2 Ferlay J, Colombet M, Soerjomataram I, et al. Cancer statistics for the year 2020: An overview. International journal of cancer 2021. 3 Mazzaferro V, Sposito C, Zhou J, et al. Metroticket 2.0 Model for Analysis of Competing Risks of Death After Liver Transplantation for Hepatocellular Carcinom a . Gastroenterology 2018;154(1):128-39. 4 Orcutt ST, Anaya DA.Liver Resection and Surgical Strategies for Management of Primary Liver Cancer. Cancer control: journal of the Moffitt Cancer Center 2018;25(1): 1073274817744621. 5 Wallace MC, Preen D, Jeffrey GP, Adams LA. The evolving epidemiology of hepatocellular carcinoma: a global perspective. Expert review of gastroenterology \& hepatology 2015;9(6):765-79. 6 Ling CQ, Fan J, Lin HS, et al. Clinical practice guidelines for the treatment of primary liver cancer with integrative traditional Chinese and Western medicine. Journal of integrative medicine 2018;16(4):236-48. 7 Yang L, Li TT, Chu YT, et al. Traditional Chinese medical comprehensive therapy for cancer-related fatigue. Chinese journal of integrative medicine 2016;22(1):67-72. 8 Wong CHL, Wong W, Lin WL, et al. Prioritizing Chinese medicine clinical research questions in cancer palliative care from patient and caregiver perspectives. Health expectations : an international journal of public participation in health care and health policy 2021;24(4):1487-97. 9 Zhao H, Miao $M$. The application characteristics of Zhongjing external prescription medicine. Saudi journal of biological sciences 2017;24(8):1903-06. 10 Pu CY, Lan VM, Lan $C F$, Lang HC. The determinants of traditional Chinese medicine and acupuncture utilization for cancer patients with simultaneous conventional treatment. European journal of cancer care 2008;17(4):340-9.

Keywords: acupuncture, External application of Chinese medicine, metaanalysis, primary liver cancer, protocol, safety, systematic review.

\section{Contributions of each author:}

Author 1 - Song Wang - The author evaluates and evaluates, designs and evaluates, negotiates and evaluates, collects data, understands the data, and writes a plan.

Email: 375141121@qq.com

Author 2 - Zhuang Xiong - The author designs evaluations, coordinates evaluations, data collection, data management, data analysis and funding.

Email: 172933813@qq.com

Author 3 - Yangyang Liu - The author coordinates the evaluation, data management, interpretation of the data, and preparation of the plan.

Email: 996439299@qq.com

Author 4 - Yan Leng - The author designs evaluations, coordinates evaluations, data management, and interprets data.

Email: 1289021445@qq.com

Author 5 - Houbo Deng - The author designs evaluations, coordinates evaluations, data management, and interprets data.

Email: 37400139@qq.com

Author 6 - Dong Shen - This author coordinates evaluation, data collection, data management, data analysis and interpretation of data.

Email: 280783305@qq.com

Author 7 - Xiangtong Meng - This author coordinates evaluation, data collection, 
data management, data analysis and interpretation of data.

Email: 838389400@qq.com

Author 8 - Tiejun Liu - The author conceives the evaluation, coordinates the evaluation, interprets the data and reviews the plan. 